\title{
Information storage capacity of incompletely connected associative memories
}

\author{
Holger Bosch ${ }^{\mathrm{a}}$, Franz J. Kurfess ${ }^{\mathrm{b}, *}$ \\ ${ }^{a}$ Department of Computer Science, University of Geneva, Geneva, Switzerland \\ ${ }^{\mathrm{b}}$ Department of Computer and Information Science, New Jersey Institute of Technology, Newark, NJ, USA
}

\begin{abstract}
In this paper, the memory capacity of incompletely connected associative memories is investigated. First, the capacity is derived for memories with fixed parameters. Optimization of the parameters yields a maximum capacity between 0.53 and 0.69 for hetero-association and half of it for autoassociation improving previously reported results. The maximum capacity grows with increasing connectivity of the memory and requires sparse input and output patterns. Further, parameters can be chosen in such a way that the information content per pattern asymptotically approaches 1 with growing size of the memory.
\end{abstract}

Keywords: Associative memory; Information storage capacity; Incomplete interconnectivity; Hetero-association; Auto-association; Hebbian learning; Retrieval strategy; Sparse coding

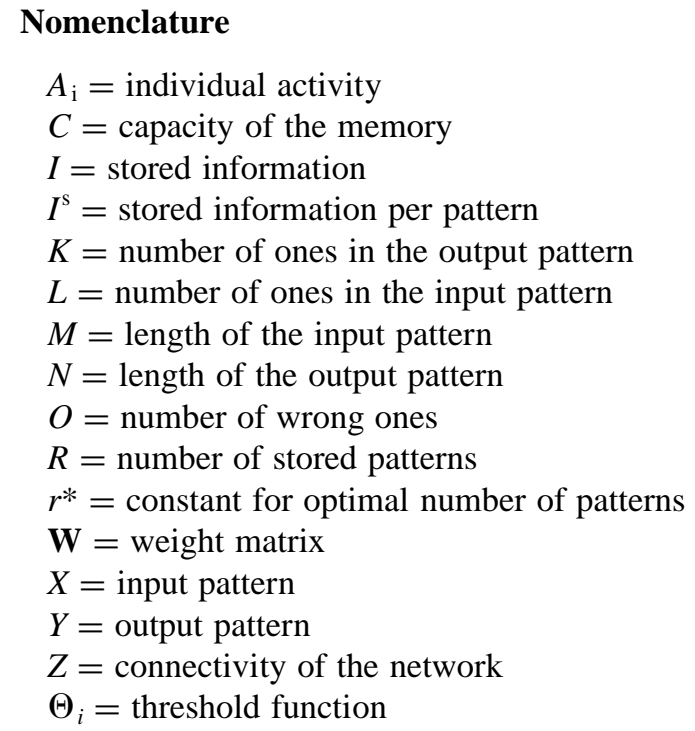

\section{Introduction}

Tasks like voice or face recognition, which are hard to realize with conventional computer systems, can benefit considerably from using content-based access techniques as

\footnotetext{
* Corresponding author. Tel: 001973596 5767; Fax: 001973596 5777; e-mail: franz@cis.njit.edu
}

offered by associative memories. This kind of memory is robust against input noise and has a practically constant retrieval time independent of the number of stored associations. Associative memories have been intensively studied in the past (Steinbuch, 1961; Kohonen, 1977; Palm, 1980; Palm et al., 1993; Willshaw, 1971 for instance), resulting in an established theory and many successful applications. Most of the models are fully connected memories, which can be applied immediately to actual digital implementations of associative memories with currently available electronic memory technology.

However, technological advances or paradigm shifts like optical or analog associative memories may lead to partially connected memories, as a result of physical and functional properties of the materials and techniques used. Hence, models for incompletely connected memories need to be considered and investigated in more depth. Another reason to study incompletely connected associative memories is their relevance for biological neural networks. The parts of the brain that exhibit functional properties of associative memory are not at all fully connected. For example, the neurons of some parts of the hippocampus are connected to not more than $5 \%$ of the neurons in their neighborhood (Amaral et al., 1990). Results from investigations concerning incompletely connected memories may lead to a better understanding of the brain, even if the considered models are far from neurobiological reality. 
One of the most important features of any memory is its storage capacity, which is the quotient between the amount of information that can be maximally stored and the size of the memory. Whereas conventional memory models achieve an optimal storage capacity, associative memories can not fully exploit their storage elements, because of the distributed representation of information, and their capacities depend on factors as their retrieval strategies for instance.

The present paper can be seen as an extension of (Palm, 1980) who studied completely connected associative memories. His work is based on a review article (LonguetHiggins et al., 1970) and a book Kohonen (1977). Palm discusses a simple binary model and reports a maximum capacity of $\ln (2) \approx 0.69$ for the hetero-associative memory and $\ln (2) / 2 \approx 0.34$ for the auto-associative memory.

Whereas an optimal retrieval strategy exists for the fully connected model, there is no such optimal method if the memory is only incompletely connected. Several strategies have been studied and compared by Buckingham and Willshaw (1993). Since the output of a memory depends highly on its retrieval strategy, the memory capacity differs for each strategy. Our study is based on one of the above methods, which has several advantages in order to achieve a high capacity. A brief comparison with other retrieval strategies is given in the Discussion.

In a more recent paper, Graham and Willshaw (1997) study the capacities of incompletely connected memories with respect to three different retrieval strategies (Buckingham and Willshaw, 1993). However, the authors use different definitions Palm (1980), for memory capacity and efficiency, since they insist upon error-free retrieval of the output pattern. In their case, capacity is defined as the maximum number of stored associations. Their results are entirely obtained through simulations on memories of considerable size and are used to estimate the capacity of the hippocampus.

In the present paper, we investigate the capacity of incompletely connected associative memories as defined in (Palm, 1980). We show that maximum capacities between 0.53 and 0.69 can be achieved depending on the level of connectivity. Further, we show that for a growing size of the memory, asymptotically all the information contained in the patterns can be stored. A similar result is presented in (Palm, 1981), which is an extension of the original result on completely connected memories (Palm, 1980). However, a different retrieval strategy from ours is used in this model, and thus the two studies are complementary to each other. Other discussions of partially connected neural associative memories can be found in (Frolov and Muravev, 1993, Hogan and Diederich, 1995 and Vogel and Boos, 1994).

In the next section, the model used as basis for our investigations is described. In Section 3, the capacity of an incompletely connected memory with fixed parameters is derived, both for the hetero-associative and the autoassociative case. In Section 4, the maximum capacities are obtained analytically by optimization of the parameters which are confirmed through numerical optimization and simulations described in Section 5. Section 6 concentrates on the information content per pattern, showing that a high information content can be achieved together with a high capacity by selecting appropriate parameter values. In Section 7, the results obtained are put into perspective, and possibilities for future work are outlined.

\section{Description of the model}

This section contains a formal description of the associative memories on which the present study is based. The hetero-associative case is described in detail; the autoassociative memory discussed in the second part differs only slightly from the hetero-associative one. Our model is similar to the one considered in (Palm, 1980). Whereas Palm's model is completely connected, ours contains an additional parameter describing the degree of connectivity between the input and the output layer.

\subsection{Hetero-associative memory}

The purpose of a hetero-associative memory is to store pairs of input and output patterns; such a pair of input and output patterns is also referred to as association. The input patterns $X^{s}, s=1 \ldots R$ and the output patterns $Y^{s}$ are binary vectors of finite dimension, i.e. $X^{s} \in\{0,1\}^{M}$ and $Y^{s} \in\{0,1\}^{N}$. Each $X^{s}$ (resp. $Y^{S}$ ) contains exactly $L$ (resp. $K$ ) ones, and a total number of $R$ associations is stored in the memory. The input layer is connected with the output layer by $Z M N$ equally distributed connections, where $Z$ describes the degree of connectivity; for a fully connected memory, $Z=1$. The binary weights of the existing connections $w_{i j} \in\{0,1\}$ are determined by a Hebbian learning rule:

$w_{i j}=\max _{s=1 \ldots R}\left(Y_{i}^{s} \otimes X_{j}^{s}\right)$

where $X \otimes Y$ is the outer product of the two vectors. The memory can be interpreted as a partially filled matrix $\mathbf{W}$ of dimension $M \times N$ with elements $w_{i j} \in\{0,1\}$ representing the weights of existing connections. For missing connections, there is no entry in the corresponding position of the matrix. For some of our considerations, it is essential to distinguish between existing connections with a weight of 0 , and missing connections.

For completely connected memories, there is an optimal retrieval strategy; for incompletely connected models no such optimal retrieval strategy exists (Buckingham and Willshaw, 1993). Several retrieval methods are studied and compared in the above paper; our approach is based on an activity-based thresholding strategy defined therein.

To retrieve the response to an input pattern $X$ the weighted sums $Y_{i}^{\prime} \sum_{j} w_{i j} X_{j}$ are calculated, where the sums are only over the existing weights $w_{i j}$. This means that input values corresponding to missing connections are not 
considered for retrieval. The final response is obtained by comparing $Y^{\prime}$ with the thresholds given by the input activities. The individual activity $A_{\mathrm{i}}$ for an output unit $Y_{i}$ is defined by $A_{i}=\Sigma_{j} X_{j}$ where the sum is again only over the existing connections. The output of the memory is then given by

$Y_{i}=\left\{\begin{array}{l}1 \text { if } Y_{i}^{\prime} \geq A_{\mathrm{i}} \\ 0 \text { else }\end{array}\right.$

An output unit is set to one if each existing connection to a one in the input has weight one. Therefore, if a learned input pattern $X^{s}$ is applied to the memory, the answer contains all the $K$ ones of its answer $Y^{s}$ but may contain $O$ additional spurious ones.

\subsection{Auto-associative memory}

The purpose of an auto-associative memory is the storage and retrieval of individual patterns, where the pattern applied to be retrieved may be incomplete or distorted by noise. In this case, input and output vectors are identical, which implies $N=M$ and $K=L$. The connections are symmetrical, i.e. $w_{i j}=w_{j i}$ if both connections exist.

\section{Storage capacity of the associative memory}

The capacity of an associative memory is defined as the ratio of the information stored in the memory and its total size:

$C=\frac{I}{Z M N}$

The size is given by the connectivity, the dimension of the input vector, and the dimension of the output vector; in conventional memory terms, it is the number of bits available for information storage. The total information stored in the memory $I$ is the sum of the individual informations $I^{s}$ stored per output pattern, $I=\sum_{s} I^{s}$. They are defined in terms of Shannon's information theory and diverge for heteroassociative and for auto-associative memories.

Since the actual contents of the memory are not known in general, the calculations are based on expected values. The expected value of the capacity $E(C)$ is given by

$E(C)=\frac{E(I)}{Z M N}$

We first study the capacity of hetero-association and then extend the calculations to the auto-associative case. In both cases, we consider memories with fixed parameters. The calculations are partly inspired by those for the completely connected model (Palm, 1980).

\subsection{Hetero-associative memory}

In the case of hetero-association, $I^{s}$ is defined as the difference between the information contained in the output pattern $Y^{s}$ and the information needed to determine this pattern given the response of the memory to $X^{s}$ :

$I^{s}=\operatorname{ld}\left(\begin{array}{c}N \\ K\end{array}\right)-\operatorname{ld}\left(\begin{array}{c}O+K \\ K\end{array}\right)$

where $\operatorname{ld}(x)$ is the logarithm base 2 of $x$ and $O$ the number of spurious ones in the output. The second term in this equation is also referred to as correction information. A lower bound for the expectation value of $I$ is given by (Palm, 1980):

$\mathrm{E}(I) \geq R \sum_{i=0}^{K-1} \operatorname{ld} \frac{N-i}{K+(N-K) \mathcal{P}-i}$

where $\mathcal{P}$ is the probability of a spurious one, or an output unit being one instead of zero. The number of spurious ones in the output is $O=(N-K) \mathcal{P}$. The determination of the probability $\mathcal{P}$ is one of the crucial points in our calculations since it is the main unknown factor in the above equation. Such a spurious one only occurs if all existing connections between the zero in the output and the ones in the input have weight one. The probability of a connection having weight zero is given by

$\operatorname{Pr}\left(w_{i j}=0\right)=\left(1-\frac{L K}{M N}\right)^{R-1} \approx \mathrm{e}^{-R \frac{L K}{M N}}$

where the approximation is tight for large numbers of sparse patterns. Unfortunately, the occurrences of ones in the same row or column are not independent since in the model discussed here there is a fixed number of ones.

For sparse input patterns and a large number of stored patterns, however, this dependence is very weak. Since the maximum capacity is reached for these parameters, the independence of the occurrences is assumed a posteriors throughout this paper. For a more detailed discussion see (Palm, 1980).

The probability $\mathcal{P}$ can therefore be approximated by

$\mathcal{P} \approx\left(1-Z \operatorname{Pr}\left(w_{i j}=0\right)\right)^{L} \approx\left(1-Z \mathrm{e}^{\left.-R \frac{L K}{M N}\right)^{L}}\right.$

since the input contains a fixed number $L$ of ones, and $\left.(1-Z) \operatorname{Pr}\left(w_{i j}=0\right)\right)$ equals the probability that a connection either does not exist or has weight one.

This concludes the calculation of the capacity of the hetero-associative memory. Eq. (3) gives an approximation for the probability of spurious ones which is used to determine a lower bound for the expected value of the stored information in Eq. (2). This in turn determines the expectation value of the memory capacity in Eq. (1).

\subsection{Auto-associative memory}

The capacity calculations for auto-associative memories depend on the determination of the stored information which is again the sum of the individual informations stored per pattern. The correction information per pattern, 
however, must be defined differently since the input pattern is identical to the output pattern and may be different from the pattern used for retrieval.

The correction information is determined incrementally, increasing the number of genuine ones and decreasing the number of spurious ones at each step. To retrieve a pattern, a single one of the output pattern is determined at each step, starting with a vector containing only zeros. A step consists of the application of the actual pattern to the memory and the determination of an additional genuine one. The sum of the information of these determinations equals the correction information. Hence the information stored in the memory is given by ${ }^{1}$

$\mathrm{E}(I) \geq R \sum_{i=0}^{K-1} \operatorname{ld} \frac{N-i}{K+\mathrm{E}\left(O_{i}\right)-i}$

where $\mathrm{E}\left(O_{i}\right)$ is the expectation value of the number of spurious ones in the $i$ th step. Note that the input pattern applied in step $i$ contains exactly $i$ ones. Similar to the hetero-association case

$$
\begin{gathered}
\mathrm{E}\left(O_{i}\right) \approx(N-K)\left(1-Z \operatorname{Pr}\left(w_{i j}=0\right)\right)^{i} \\
=(N-K)\left(1-Z\left(1-\frac{K^{2}}{N^{2}}\right)^{R-1}\right)^{i}
\end{gathered}
$$

In order to calculate the memory capacity for the auto-associative case, we determined the correction information per pattern in a different way; the rest of the calculations are identical to the hetero-associative case.

\section{Maximum capacity of associative memories}

The optimization is done in order to obtain maximal capacities for all levels of connectivity. First, the capacity is maximized for hetero-association and the calculations are presented in detail. In a second step, the optimization is extended to auto-association.

\subsection{Hetero-associative memory}

The optimization is based on the equations derived in the previous section, i.e. Eqs. (1)-(3). Starting from Eq. (2), a simpler lower bound for the expectation value of the stored information $\mathrm{E}(I)$ is derived. Since $(A-i) /(B-i) \geq A / B$ for $A \geq B$, and $N \geq K+(N-K) \mathcal{P}$, we obtain

$$
\begin{aligned}
\mathrm{E}(I) & \geq R K \operatorname{ld} \frac{N}{K+(N-K)-\mathcal{P}} \geq R K \operatorname{ld} \frac{1}{\mathcal{P}+\frac{K}{N}} \\
& =R K\left(\operatorname{ld} \frac{1}{\mathcal{P}}-\operatorname{ld} d\right)
\end{aligned}
$$

where $d=(P+K / N) / \mathcal{P}$ is the ratio between the total number of ones and the number of spurious ones in the output. Substitution of the probability $\mathcal{P}$ by Eq. (3) yields

$$
\begin{aligned}
& \mathrm{E}(I) \geq R K\left(\operatorname{ld} \frac{1}{\left(1-Z \mathrm{e}^{-R \frac{L K}{M N}}\right) L}-\mathrm{ld} d\right) \\
& =R K L\left(\operatorname{ld} \frac{1}{\left(1-Z \mathrm{e}^{\left.-R \frac{L K}{M N}\right) L}\right.}-\frac{\mathrm{ld} d}{L}\right)
\end{aligned}
$$

Writing $R=r \frac{M N}{L K}$ for the number of stored patterns, the expectation value of the total capacity is given by:

$$
\mathrm{E}(C) \geq \frac{r}{Z}\left(\operatorname{ld} \frac{1}{1-Z \mathrm{e}^{-r}}-\frac{\mathrm{ld} d}{L}\right)
$$

As the arguments of both logarithms are larger than 1, the first term increases the capacity and the second decreases the capacity. Whereas the first term depends only on the connectivity $Z$, the second one depends among others on the parameter $L$. Its influence can be limited by increasing the number of ones in the input, as the ratio $d$ can be supposed to be bounded. However, since $L<M$ is required in order to avoid overloading of the memory, better results are only achieved for large memories which allow large values for $L$. This is especially important for memories of low connectivity since the first term approaches 0 in this case.

Ignoring therefore the term $l d d / L$ in Eq. (6), an approximation of the capacity is given by

$\mathrm{E}(C) \approx \frac{r}{Z}\left(\operatorname{ld} \frac{1}{1-Z \mathrm{e}^{-r}}\right)$

which depends only on the connectivity $Z$ and the above introduced parameter $r$.

First, let us consider the case of vanishing connectivity, i.e. $Z \rightarrow 0$. For fixed $r$, the storage capacity is given by

$\lim _{Z \rightarrow 0} \mathrm{E}(C) \frac{r \mathrm{e}^{-r}}{\ln 2}$ (de $1^{\prime}$ Hospital)

$r \mathrm{e}^{-r}$ reaches its maximum for $r=1$, which implies

$\lim _{Z \rightarrow 0} \mathrm{E}(C)=\frac{1}{\mathrm{eln} 2} \approx 0.5307$

for the maximum capacity of vanishing connectivity.

Next, fixing $Z \epsilon] 0,1]$, a numerical optimization of the parameter $r$ shows a maximum capacity between 0.5307 and 0.69 , where the maximum capacity increases with growing connectivity (Fig. 1). The optimal number of patterns for the maximum capacity is given by $R^{*}=r^{*}(Z) M N /$ $L K$ where $r^{*}(Z)$ is a decreasing function between $r^{*}(0.0) \approx$ 1 and $r^{*}(1.0) \approx 0.7$. Note that the only condition imposed on the parameters $K$ and $L$ is that they are considerably smaller than $N$ and $M$. Therefore, the maximum capacity should be feasible for a large range of values for $K$ and $L$, supposed 


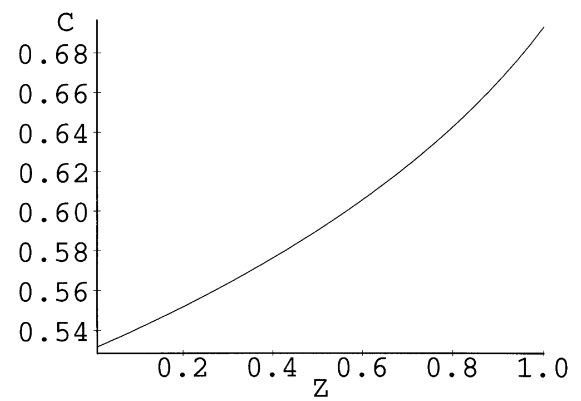

Fig. 1. Maximum capacity of the incompletely connected heteroassociative memory as a function of the connectivity $Z$. It is achieved for sparse input and output patterns and an appropriate number of stored associations. The maximum capacities as well as the optimal parameters were derived analytically.

that the number of stored associations is close enough to the optimal value $R^{*}=r^{*}(Z) M N / L K$.

Numerical optimization and simulations show that, even for low connectivity, the maximum capacities obtained from the above approximations can be achieved for appropriate choices of parameters (see next section).

\subsection{Auto-associative memory}

The maximum capacity for auto-associative memories is calculated for an arbitrary degree of connectivity. The optimization is based on Eqs. (4) and (5) describing the capacity of a memory with fixed parameters (see earlier). The method is similar to the optimization of hetero-associative memories and is therefore only outlined.

The lower bound for the expectation value of the stored information in Eq. (5) can be further approximated by

$\mathrm{E}(I) \geq \frac{1}{2} R(K-1)(K-2) \mathrm{ld}\left(\frac{1}{1-Z \mathrm{e}^{-R \frac{K^{2}}{N^{2}}}}\right)-R \sum_{i=0}^{K-1} \mathrm{ld} d_{i}$

where $d_{i}$ is the ratio between the total number of ones and the number of spurious ones in the output at step $i$. Finally, with $R=r \frac{N^{2}}{K^{2}}$ we obtain

$\mathrm{E}(C) \geq \frac{r}{2 Z} \operatorname{ld} \frac{1}{1-Z \mathrm{e}^{-r}}-\frac{1}{Z K^{2}} \sum_{i=0}^{K-1} \operatorname{ld} d_{i}$

Considering that only half of the symmetrical matrix is necessary the result is the same as in the case of heteroassociation and the same optimization yields values between 0.26 and 0.34 for the maximum capacity.

The maximum capacities for incompletely connected memories derived in this section are close to the capacity of fully connected memories, making their usage equally interesting to the usage of fully connected models. Although optimization does not account for low retrieval error, theoretical considerations show that this can be achieved asymptotically for large memories (cf. Section 6).

\section{Numerical optimization and simulations}

In order to verify the values for the maximum capacity derived above, numerical optimizations and simulations have been performed. Both are restricted to heteroassociative memories, since the calculations for the autoassociative memories are based on similar approximations.

\subsection{Numerical optimization}

The optimizations were done over a wide range of the free parameters in order to ensure maximal capacities. For each set of fixed parameters, the expectation value of the memory capacity was computed using Eqs. (1)-(3). The fixed parameters were the connectivity $Z$ and the size of the memory, i.e. $M$ and $N$. The remaining free parameters used for the optimization were the number of stored patterns $R$ and the number of ones in the input and output patterns $L$ and $K$. The optimizations were done for two sizes of the memory, i.e. $N=M=100$ and $N=M=1000$.

For both cases, the maximal capacities were determined for any level of connectivity and the obtained results confirm exactly the theoretical predictions of the previous section. The maximal capacities yield values between 0.53 and 0.69 and increase with growing connectivity.

\subsection{Simulations}

Since the formulas used in the numerical optimization are not absolutely exact and approximate a lower bound instead of the capacity, simulations were performed to further confirm our theoretical results.

Again, the computations were restricted to the heteroassociative case and the two memory sizes $N=M=100$ resp. $N=M=1000$. The numbers of ones in the patterns were fixed at $K=L=3$ resp. $K=L=4$. For the number of stored patterns, the estimations of the previous section $R^{*}=$ $r^{*}(Z)[(M N) /(L K)]$ were used. Several associative memories were built for each of 20 different levels of connectivity, ranging from $Z=0.05$ to $Z=1.00$. A further condition was that any two input patterns were different, i.e. $\forall i, j: X_{i} \neq Y_{i}$. This condition was neglected in the above calculations (the patterns were supposed to be independent), but this is of no great importance if $R \ll M^{L}$ is guaranteed. This is the case for $L \geq 3$ and $N \approx M$ since the number of patterns we are interested in is $R \approx N M$.

Fig. 2 shows the results of the simulations of the heteroassociative memories. The capacities of the memories of size $N=M=100$ are not yet optimal (a) whereas the capacities achieved by the networks of size $N=M=1000$ match almost perfectly the theoretically derived values (b).

Note that no optimization of the parameters was done besides the theoretical optimization of $r^{*}(Z)$. The results show clearly that the values theoretically derived in the previous section can be achieved by memories of realistic sizes. 

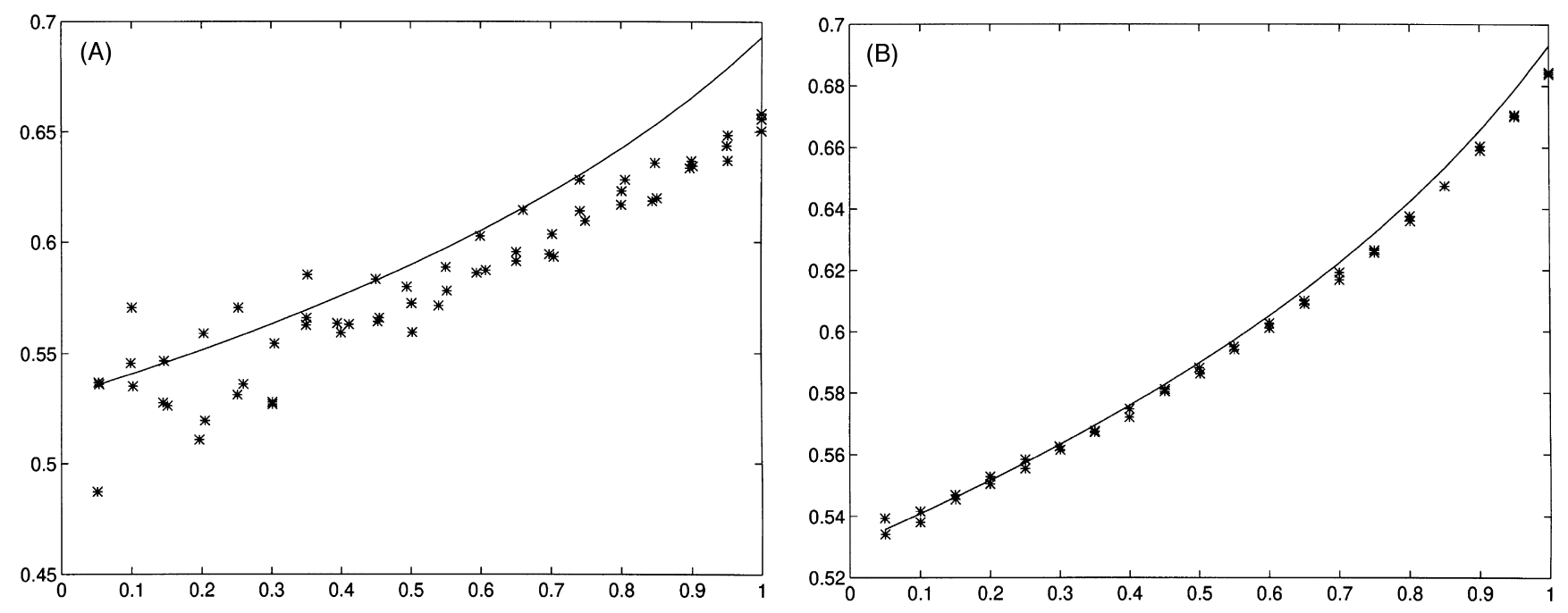

Fig. 2. Maximum capacity of the hetero-associative memory as a function of the connectivity $Z$. The continuous curves represent the analytically predicted values (cf. Fig. 1), whereas discrete points are capacities obtained from simulations. The values shown in (a) resp. (b) were obtained from memories of sizes $M$ $=N=100$ resp. $M=N=1000$.

\section{Information content per pattern}

The above optimizations of the capacity do not take into consideration the accuracy of the retrieved output patterns. Nevertheless, information content is one of the crucial criteria for the quality of a memory and should be achieved besides high capacity by any useful memory.

The information content $P$ for an individual pattern $I^{S}$ is defined as the ratio between the stored information of the pattern itself and the information contained in the output pattern $\bar{I}^{s}=\bar{I}$, where

$\bar{I}=\operatorname{ld}\left(\begin{array}{l}N \\ K\end{array}\right) \approx K \operatorname{ld} N$

in the case of sparsity. The information content $P$ can also be defined for the entire memory as the mean of the information contents of the individual patterns. The expectation value of the information content is given by:

$\mathrm{E}(P)=\frac{E\left(I_{s}\right)}{\bar{I}} \approx \frac{E\left(I_{s}\right)}{K \operatorname{ld} N}=\frac{\mathrm{E}(I)}{R K \operatorname{ld} N}$

The last equality holds because $R K 1 \mathrm{~d} N$ is the total information of the output patterns. Thus, the condition $R K \operatorname{ld} N=$ $\mathrm{E}(I)$ guarantees that roughly all information of the patterns is stored, i.e. $P \cong 1$. In the case of maximum capacity, the total information stored in the memory is given by $\mathrm{E}(I)=-R K L\left(\operatorname{ld}\left(1-\mathrm{Ze}^{-\mathrm{r}^{*}(\mathrm{Z})}\right)-\mathrm{ld} d / L\right)$ (see above) which provides the following condition for $L$ :

$L=\frac{\operatorname{ld} N+\operatorname{ld} d}{-\operatorname{ld}\left(1-Z \mathrm{e}^{-r *(Z)}\right)} \approx \frac{1}{\operatorname{ld} \frac{1}{1-Z \mathrm{e}^{-r *(Z)}}} \mathrm{ld} N$

where the factor preceding $\mathrm{ld} N$ increases with decreasing connectivity. Low connectivity requires a larger number of input ones than high connectivity to achieve an accurate result. The reason is that a minimum level of activity must be present in order to avoid too many spurious ones. With the value for $L$ given in Eq. (8), the activity is almost independent of the connectivity $Z$.

This condition on $L$ is not necessarily fulfilled by the above determination of maximum capacity. In particular, the number $L$ of ones in the simulations of the previous section does not depend on the level of connectivity but is kept constant $(L=3$ for $N=M=100$ and $L=4$ for $N=$ $M=1000)$. In this case, the information content $P$ depends linearly on the level of connectivity $Z$, implying that $P$ asymptotically vanishes if $Z$ approaches 0 . This comes from the fact that less and less output units are connected to ones in the input with the implication that these output units are set to one.

Recall that high capacity demands sparse input patterns, i.e. $L \ll M$. If the input and output dimensions are roughly of the same order of magnitude, which is mostly the case, it also implies $L \ll N$. Thus, in order to obtain a high information content and an optimal capacity, it is necessary that the value of $L$ given in Eq. (8) verifies this sparsity condition. For high connectivity the factor preceding $\operatorname{ld} N$ is small enough that $L$ $\ll M$ is verified even for relatively small memories. For decreasing connectivity this factor becomes more important and $L \ll M$ is only given for memories of large sizes. However, if the connectivity $Z$ is fixed, the factor remains constant and the sparsity condition is asymptotically verified for memories of growing sizes, since

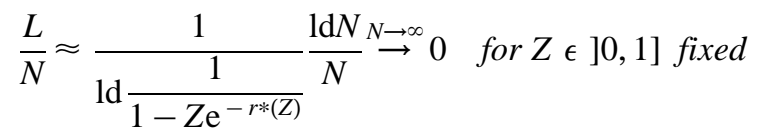

The dependency of the capacity and the information content on the number of ones in the input pattern is illustrated in 
Fig. 3(a). The connectivity is fixed at $Z=0.5$ and the number of patterns is optimized to obtain maximum capacity. The increasing function represents the information content per pattern, confirming that a high activity is necessary for an accurate result. The capacity decreases with the number of input ones since the network is of relatively small size $(N=M=1000)$ leading to a fast overloading of the memory for increasing values of $L$.

Fig. 3(b) shows the capacity and the information content for an increasing number of patterns $R$ with connectivity fixed at $Z=0.5$. The capacity increases first with the number of patterns before it begins to decrease when too many ones in the matrix are stored. The information content is a steadily decreasing function as expected.

In conclusion, both maximum capacity and optimal information content are feasible at the same time for any degree of connectivity. Numerical evaluations for very large memories of the Eqs. (1)-(3) describing the capacity and the information content confirm the analytical result, i.e. $P \approx 1$ and $C \epsilon[0.53,0.69]$, depending on the connectivity $Z$ (data not shown).

\section{Discussion}

The core aspect of this paper is the study of memory capacities and information content for incompletely connected associative memories. Theoretical investigations show that the maximum capacities range from 0.53 to 0.69 , depending on the connectivity of the network. These capacities can be obtained for sparse input and output patterns and appropriate numbers of stored associations. Additionally, for large memories more restricted choices of parameters guarantee a high information storage per pattern which asymptotically approaches 1 . These theoretical considerations have been confirmed by numerical simulations.

Our results have been obtained for one particular model of binary associative memory with one particular out of several possible retrieval strategies. It should be asked if other models resp. other retrieval strategies are likely to have similar properties regarding their capacities and information contents.

In general, associative memories are not restricted to binary or discrete models and might also rely on patterns and weights of continuous values. But it is more difficult, if not impossible, for these models to quantify the information contained in the memory, a pattern or the information necessary to correct an erroneous answer and the problem of their capacities might thus be hard to define.

But even the restriction to binary models yields several alternatives. For instance, the patterns can be coded through and the weights may assume one of the values \pm 1 instead of zero or one. Models may be based on other learning rules to determine the weights of the connection and on different retrieval strategies as mentioned above. Furthermore, the kind of incomplete connectivity may also differ. A
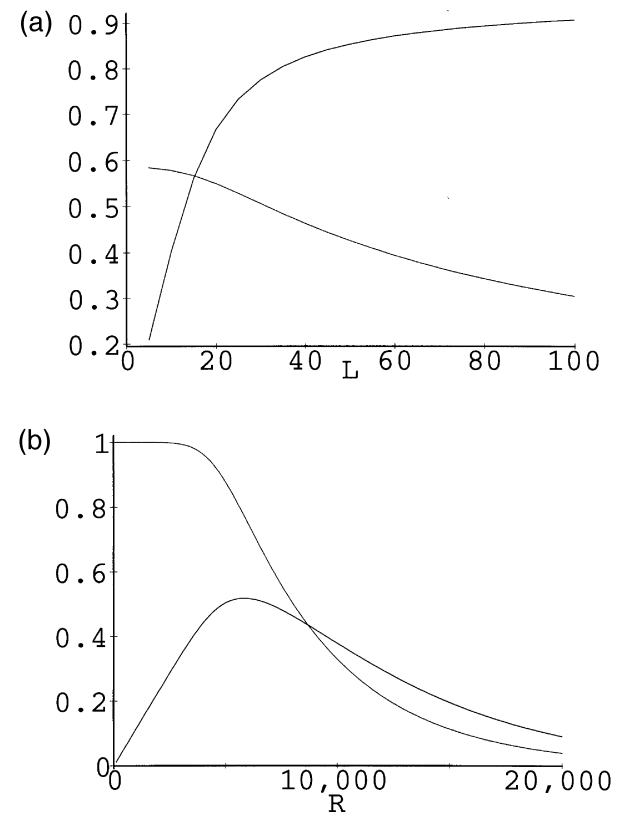

Fig. 3. (a) Maximum capacity (decreasing curve) and information content per pattern of the hetero-associative memory as functions of the number $L$ of ones in the input patterns. The fixed parameters are $N=M=1000, K=$ 10 and $Z=0.5$ whereas the number of stored patterns is optimized. The information content increases with the number of ones since an increased activity improves the correctness of the answer. The capacity is a decreasing function since a large value of $L$ leads to overloading the memory. (b) Capacity and information content per pattern (decreasing curve) as functions of the number of stored patterns $R$. The fixed parameters are $N=M=1000, Z=0.5, K=10$ and $L=30$. For small $R$, the memory is able to store the entire information of the associations. Thus, the information content remains close to 1 and the capacity increases linearly with $R$. As soon as overloading occurs, the information content starts to decrease and the capacity reaches its maximum shortly afterwards. The calculations are based on Eqs. (1)-(3).

connection may not be missing but existing and permanently be one or zero.

However, some general remarks concerning their capacities may apply to most of the binary models of incompletely connected associative memories, at least to those whose patterns and weights have values zero or one.

First of all, one important characteristic is whether the number of ones in the patterns is fixed. If this is not the case, some patterns may contain a high number of ones and any hebbian learning will lead to a fast overloading of the memory, decreasing its capacity.

Another reason to fix the number of ones in the output pattern is that less correction information might be necessary to obtain the correct answer. Under the further condition that only spurious ones or spurious zeros occur, and not both of them at the same time, the number of erroneous output units can be calculated without any further information. If both types of errors occur, an additional spurious one and an additional spurious zero will not change the number of zeros and ones in the output. This latter case was studied in (Palm, 1981), who investigated a model with adaptive 
threshold, leading to spurious ones and zeros. Palm established a lower bound of 0.05 for the capacity of a heteroassociative memory. This bound is considerably lower than those presented in this paper, illustrating that it is more difficult to detect spurious zeros and spurious ones at the same time.

The possibility that only spurious zeros and no spurious ones occur is not a very realistic problem since in most models the overloading of the memory leads inevitably to spurious ones in the answer. If only spurious ones occur and the patterns are sparse, i.e. they contain only a few ones, then the correction information can be greatly reduced as in our model. This stems from the fact that the number of spurious units is kwown and that, under the condition of sparse output patterns, it costs less to determine a unit among a few others than among a large set of units.

The above considerations concern fully connected as well as incompletely connected models. In the latter case, additional erroneous output units may occur through missing connections. Again, additional spurious ones reduce the capacity less than spurious zeros do, for the same reason that a mixture of both types of errors implicates that the number of errors can not be determined any more without further information.

Further work investigates the memory capacity for other retrieval strategies, modified learning rules or different architectures of incompletely connected associative memories. In collaboration with other researchers, implications for manufacturing large associative memories with possible imperfections are being explored. This aspect can be relevant for yield improvements of high capacity memory chips, where the likelihood of imperfections increases substantially with the size of the chip. Mutual benefits are also expected from a collaboration with neuroscientists, comparing the properties of our abstract model with biological neural networks that exhibit behavior similar to associative memories.

\section{Acknowledgements}

We would like to thank the anonymous referees for their suggestions on improvements, in particular the refined result in Eq. (7).

\section{References}

Amaral D. G., Ishizuka N., \& Claiborne B. (1990). Neurons, numbers and the hippocampal network. Progress in Brain Research, 83, 1-11.

Buckingham J., \& Willshaw D. (1993). On setting unit thresholds in an incompletely connected associative net. Network, 4, 441-459.

Frolov A., \& Muravev I. (1993). Informational characteristics of neural networks capable of associative learning based on Hebbian plasticity. Network, 4, 495-536.

Graham B., \& Willshaw D. (1997). Capacity and information efficiency of the associative net. Network, 8, 35-54.

Hogan, J., \& Diederich, J. (1995). Random neural networks of biologically plausible connectivity. Technical Report, Queensland University of Technology, Australia.

Kohonen, T. (1977). Associative memory. New York: Springer.

Longuet-Higgins H. C., Willshaw D. J., \& Bunemati O. P. (1970). Revue Biophysique, 3, 223.

Palm G. (1980). On associative memory. Biological Cybernetics, 36, 1931.

Palm G. (1981). On the storage capacity of an associative memory with randomly distributed storage elements. Biological Cybernetics, 39, $125-127$.

Palm G., Kurfess F., Schwenker F., \& Strey A. (1993). Neural associative memories. Technical Report, Universität Ulm, Germany.

Steinbuch K. (1961). Die Lernmatrix. Kybernetik, 1, 36.

Vogel D., \& Boos W. (1994). Minimally connective, auto-associative, neural networks. Connection Science, 6 (4), 461-469.

Willshaw D. (1971). Models of distributed associative memory. Ph.D. Thesis, University of Edinburgh. 